\title{
Environmentally responsive temperature instability in pediatric spinal cord injury
}

\author{
DE McLean*,1, J Kearney ${ }^{2}$ and MF Cawley ${ }^{3}$ \\ ${ }^{1}$ Division of Physical Medicine and Rehabilitation, The Glenrose Rehabilitation Hospital, The University of Alberta, \\ Canada; ${ }^{2}$ Division of Pediatric Rehabilitation, Department of Risk Management, Alfred I. DuPont Institute, \\ Wilmington, Delaware, USA and ${ }^{3}$ Avon Grove Pediatric Associates, West Grove, Pennsylvania, USA
}

\begin{abstract}
Objective: To study the frequency and clinical implications of environmentally responsive temperature instability in hospitalized pediatric patients with spinal cord injury (SCI).

Setting: A tertiary level SCI rehabilitation unit located in a free standing children's hospital in Wilmington, DE, USA.

Study Design: Temperature data and corresponding clinical responses were collected prospectively between January 1991 and June 1993. Fifty-four consecutive patients with SCI levels at or above T6 were admitted to the pediatric spinal cord injury rehabilitation unit over that time (4059 SCI days).

Methods: Hypothermic events were defined as oral temperatures less than $35.0^{\circ} \mathrm{C}$ or rectal temperatures less than $35.6^{\circ} \mathrm{C}$. Hyperthermic events were defined as oral temperatures greater than $38.0^{\circ} \mathrm{C}$ or rectal temperatures greater than $38.4^{\circ} \mathrm{C}$. The events and the clinical responses were reviewed retrospectively and were used for subsequent analysis if there was evidence of clinical response to environmental manipulation within 4 hours of case identification, and other potential etiologies of temperature fluctuation could be excluded.

Results: Sixty-five events of hypothermia $(1.60 \%)$ and 14 events of hyperthermia $(0.34 \%)$ were analyzed. Twelve patients $(22 \%)$ accounted for all 79 events. Subjects with environmentally responsive temperature instability were more recently injured $(P<0.001)$, had longer lengths of stay $(P<0.001)$ and were more likely to be ventilator dependent $(P<0.002)$ than those who did not have environmentally responsive temperature instability. There was no significant difference between the two groups in age, gender, level or etiology of the SCI. There were no adverse clinical outcomes as a result of the environmentally responsive temperature instability.

Conclusions: Environmentally responsive temperature instability affects a select subset of pediatric aged spinal cord injured persons. Early recognition of the potential contribution of the environment to temperature fluctuation in this group has led to the successful utilization of a temperature instability protocol on our SCI unit.
\end{abstract}

Keywords: hypothermia; hyperthermia; pediatric spinal cord injury

\section{Introduction}

Temperature instability as a result of autonomic nervous system dysfunction is a known complication of spinal cord injury (SCI). ${ }^{1,2}$ Case reports of potentially life threatening episodes of hypothermia in adults with tetraplegia have been reported. ${ }^{3,4}$ Sugarman described persistent fevers in two cases of recently injured individuals with tetraplegia in whom no infectious or other causes for the fever could be identified other than the cord injury. ${ }^{5}$ Beraldo and others reported on eight patients with SCI in whom

*Correspondence: Dr DE McLean, Division of Physical Medicine and Rehabilitation, The Nova Scotia Rehabilitation Centre Site, Dalhousie University, Queen Elizabeth II Health Sciences Centre, 1341 Summer Street, Halifax, Nova Scotia, Canada B3H 4K4 unexplained fevers were observed to resolve spontaneously. ${ }^{6}$ To our knowledge there has been no report of similar cases in children or adolescents with SCI.

Infection is the most common cause of pyrexia in SCI. ${ }^{6-8}$ However, non-infectious cases of temperature instability, causing both hypo and hyperthermic events, have also been reported in persons with cervical and high thoracic cord lesions. ${ }^{3-8}$ This type of temperature instability is presumed to result from a combined effect of autonomic nervous system dysfunction, ${ }^{1-8}$ environmental conditions ${ }^{5}$ and pharmacologic factors. $^{5,9}$ In persons with and without spinal cord lesions, thermoregulation is primarily achieved through the hypothalamus and its pathways. ${ }^{10,11}$ The presence of a cord lesion, particularly at or above the level of 
T6, impairs a person's ability to maintain homeothermia, because the neural connections between the hypothalamus and the periphery are disrupted. ${ }^{10}$

The Risk Management Department in our hospital identified hypo and hyperthermic events in pediatric patients with SCI as quality assurance (QA) parameters in need of tracking. The purpose of the QA tracking was to determine the potential risk, if any, of adverse patient outcomes as a result of environmentally induced body temperature fluctuations in this population. The purpose of this paper is to identify the frequency of environmentally responsive temperature instability in hospitalized, pediatric patients with SCI, as well as to characterize clinically those most likely to experience this phenomenon. We hypothesized that there are no specific clinical indicators to discriminate between non-environmentally responsive and environmentally responsive temperature instability in children or adolescents with spinal cord injuries. We predicted that seasonal variations in external temperatures might affect the rate of episodic hypo and hyperthermia events in our subjects, despite the presence of a computer operated environmental control system in the hospital.

\section{Methods}

Potential subjects included all consecutive pediatric admissions to the SCI Rehabilitation Unit between January 1991 and June 1993. Subjects were required to have sustained a spinal cord level of injury at or above T6, resulting in tetra or para, plegia or paresis. ASIA impairment scores were used to classify lesions as complete or incomplete ${ }^{12}$ since persons with complete cord syndromes were expected to be more likely to experience the thermoregulatory complications of a SCI. No attempt was made during the study period to address the different medication profiles that existed among the subjects. Persons for whom a cause for the temperature instability could be clinically determined were excluded from the data analysis.

Cases of hypothermia were defined as oral temperature measurements less than $35^{\circ} \mathrm{C}$, or rectal temperature measurements less than $35.6^{\circ} \mathrm{C}$. $\mathrm{Hy}$ perthermia was defined as an oral temperature greater than, or equal to, $38^{\circ} \mathrm{C}$, or a rectal temperature measurement greater than $38.4^{\circ} \mathrm{C}$. These definitions were previously determined by the Risk Management Department, and were not altered for the purpose of this analysis. Temperatures were routinely measured on all subjects with SCI at least twice per day. Additional documentation of temperature was performed by nursing staff at their discretion if the subject reported symptoms of potential temperature instability such as chills, sweats or general malaise.

Personnel of the Risk Management Department 'flagged' all potential cases of environmentally responsive hypo and hyperthermia during the study period. The clinical care team involved in the management of each patient analyzed all cases within
$4 \mathrm{~h}$ of case identification. The nursing staff was instructed to initiate a specific temperature instability protocol upon recognition of a hypo or hyperthermic patient with SCI; this protocol is outlined below. If the patient failed to respond to the protocol, physical examinations, laboratory tests and diagnostic workups were begun according to the clinical indications of each case. In general, work-ups included urinalyses, urine cultures and sensitivities, complete blood counts with differentials, tracheal secretions for gram stain and culture in ventilated patients, and blood cultures. In addition, a chest X-ray was requested if clinically warranted. If the physical examination and laboratory work-up failed to reveal a source of infection or any other cause of temperature instability, an environmentally induced case of hypo or hyperthermia was recorded.

\section{Temperature instability protocol}

Upon recognition of a temperature as previously defined, nursing staff was asked to initiate the 'temperature instability' protocol. The patients' temperature, the room temperature, the temperature of the ventilator cascade (where applicable) and the outdoor environmental temperature were recorded. In cases of individuals with hypothermia, nurses were asked to increase room temperature where this was feasible, provide warming blankets and/or a stockinette cap to the patient, and increase the ventilator cascade temperature to $30-32^{\circ} \mathrm{C}$, if necessary. For patients with hyperthermia, nurses not only removed bedclothes and the outer layers of the patients' personal clothing as appropriate, but also decreased room temperature and ventilator cascade temperature as necessary. No anti-pyretics were administered during this period. Responses to the nursing interventions were documented through follow-up temperatures. If, within $4 \mathrm{~h}$, the trend in the temperature change was not occurring in the desired direction, the medical staff was notified and a diagnostic work-up was initiated, as outlined above.

\section{Statistics}

A descriptive analysis of patient characteristics is provided. A comparison of attributes between those who demonstrated environmentally responsive temperature instability, and those who failed to demonstrate the same phenomenon was performed using Students' $t$-tests and Chi-square analyses. A $P$-level of $<0.05$ was considered to be statistically significant.

\section{Results}

Hypothermic and hyperthermic events

Over the 30-month study period, there were 72 consecutive patients admitted with spinal cord injuries at a level of T6 or above. Fifty-four different patients accounted for the 72 admissions, and the total number 
of SCI days was 4059. During this time, 65 events of environmentally responsive hypothermia and 14 events of environmentally responsive hyperthermia were documented, representing overall rates of $1.60 \%$ and $0.34 \%$ respectively. The average temperature of the hypothermic events was $35.2^{\circ} \mathrm{C}$, with a median temperature of $35.5^{\circ} \mathrm{C}$, and a range of $33.0-35.5^{\circ} \mathrm{C}$. The average temperature of the recorded hyperthermic events was $39.0^{\circ} \mathrm{C}$, with a median temperature of $39.0^{\circ} \mathrm{C}$, and a range of $38.0-40.4^{\circ} \mathrm{C}$ (Table 1). The average duration of hypothermic events was $5.2 \mathrm{~h}$, compared with an average duration of $8.5 \mathrm{~h}$ for the hyperthermic events.

Of the 54 patients involved, $12(22 \%)$ were responsible for the total 79 recorded events. One patient, a ventilator dependent child with C2 ASIA A tetraplegia, individually accounted for 34 episodes of hypothermia and eight episodes of hyperthermia over a 6 month admission. Forty-two (78\%) of the 54 patients admitted did not have any environmentally induced hypothermic or hyperthermic events during their hospitalizations.

Age

The average age of the group of subjects with environmentally induced temperature instability was 11.9 years, with a range between 5.5 and 17.6 years. In comparison, the average age of those admitted over the same time period, without experiencing any unexplained hypothermic or hyperthermic events was 11.8 years, with a range between $2.4-20.3$ years. This age difference between groups was not statistically significant.

\section{Gender}

Eleven $(92 \%)$ of the subjects with environmentally induced temperature instability were male, and one $(8 \%)$ of the subjects was female. In the population without any environmentally induced temperature instability, the sex ratio was more evenly distributed with $21(50 \%)$ males and $21(50 \%)$ females.

\section{Diagnoses}

Diagnoses in the study group of 12 patients were all cases of traumatic SCI. Two subjects (17\%) had paraplegia with ASIA A lesions at T5 and T6 respectively. The remaining ten subjects $(83 \%)$ were

Table 1 Recorded events

\begin{tabular}{lcr}
\hline & $\begin{array}{c}\text { Hypothermic } \\
\text { events } \\
(\mathrm{n}=65)\end{array}$ & $\begin{array}{c}\text { Hyperthermic } \\
\text { events } \\
(\mathrm{n}=14)\end{array}$ \\
\hline Mean temperature & $35.2^{\circ} \mathrm{C}$ & $39.0^{\circ} \mathrm{C}$ \\
Median temperature & $35.5^{\circ} \mathrm{C}$ & $39.0^{\circ} \mathrm{C}$ \\
Temperature range & $33-33.5^{\circ} \mathrm{C}$ & $38.0-40.4^{\circ} \mathrm{C}$ \\
\hline
\end{tabular}

tetraplegic or tetraparetic, and all were ventilator dependent. Eleven $(92 \%)$ of the subjects had ASIA A complete injuries, and one $(8 \%)$ was ASIA C incomplete. Four $(33 \%)$ of the subjects had associated traumatic brain or brain stem injuries.

Of the 42 patients who did not have any recorded hypo or hyperthermic events during their hospitalization, diagnoses included $37(88 \%)$ cases of traumatic SCI, four $(10 \%)$ cases of SCI due to tumor, one $(2 \%)$ case of high cervical cord injury following an episode of pneumococcal meningitis, and one $(2 \%)$ case of SCI in a patient with Coffin-Lowry Syndrome. Thirty-eight patients $(90 \%)$ in this group were tetraplegic or tetraparetic, with $36(85 \%)$ being ASIA A complete. Thirteen $(31 \%)$ of the patients were ventilator dependent, although two $(15 \%)$ of these patients required night-time ventilation only. Two patients $(4.8 \%)$ had associated brainstem injuries.

\section{Length of time since injury/illness}

The average length of time since injury or illness in the subjects who had documented events of environmentally responsive hypo or hyperthermia was 7.3 months, with a range of 10 days to 11.5 months. The average time since injury or illness in those who did not have recorded events of environmentally induced temperature instability over the study period was 22.0 months, with a range of 13 days to 160 months. This difference in length of time since injury or illness between the two groups is statistically significant at a level of $P<0.001$. (Table 2)

\section{Lengths of stay ( LOS}

The average LOS in subjects with documented events of environmentally responsive temperature instability averaged 113 days, with a range between 22 and 190 days. The LOS for patients who did not have environmentally responsive temperature instability events recorded was 48 days, with a range between 1 and 180 days. This difference in LOS between the two groups is statistically significant at a level of $P<0.001$. (Table 2) These statistically different findings in time

Table 2 Clinical characteristics of subjects

\begin{tabular}{cccc}
\hline & $\begin{array}{c}\text { Subjects } \\
\text { with events } \\
(\mathrm{n}=12)\end{array}$ & $\begin{array}{c}\text { Subjects } \\
\text { without } \\
\text { events } \\
(\mathrm{n}=42)\end{array}$ & $\begin{array}{c}\mathrm{P} \text { Value } \\
\text { (Corr) }\end{array}$ \\
\hline $\begin{array}{c}\text { Average time from } \\
\text { disability onset } \\
\text { (months) (range) }\end{array}$ & $\begin{array}{c}7.3 \\
(10 \text { days }-\end{array}$ & $\begin{array}{c}22.0 \\
(12 \text { days }-\end{array}$ & 0.001 \\
$\begin{array}{c}\text { Average length of } \\
\text { stay (days) (range) }\end{array}$ & $\begin{array}{c}160 \text { months }) \\
48\end{array}$ & 0.001 \\
$\begin{array}{c}\text { Ventilator } \\
\text { dependency }\end{array}$ & $\begin{array}{c}113 \\
\text { (22 days) }\end{array}$ & $\begin{array}{c}(1-180 \text { days }) \\
13\end{array}$ \\
\hline
\end{tabular}


since injury or illness and LOS are likely a reflection of the fact that the majority of subjects in our series with environmentally induced temperature instability were patients with newly acquired SCI.

\section{Clinical sequelae}

In two cases of individuals with hypothermia, diminished level of consciousness and symptomatic bradycardia necessitated transfer of the subject from the Rehabilitation Unit to the Pediatric Intensive Care Unit (PICU). One case of an individual with hyperthermia, also resulted in an unexpected transfer from the Rehabilitation Unit to the PICU. In this latter case, the patient was started on TrimethoprimSulfamethoxazole on day 4 of his symptoms of temperature instability for a urinary tract infection due to Enterococcus sp. However, he continued to demonstrate decreased level of arousal and hypotension, and 14 days later significant uncontrolled detrusor contractions were recorded on cystometrogram tracings. In retrospect, this detrusor instability was felt to have been the major contributing factor to the patient's autonomic instability, and this patient was excluded from the case series. The patient's autonomic symptoms eventually responded favorably to an increase in the dose of oxybutinin.

It must be noted that five other individuals with hyperthermic events, and one other individual with a hypothermic event, were subsequently excluded from the data analysis because a potential infectious etiology was subsequently identified that could account for the patients' temperature instability. All six patients had positive culture reports and were placed on antibiotics within $48 \mathrm{~h}$ of case identification. Four patients had urinary tract infections, one had Clostridium difficile isolated from the stool, and one had a case of culture proven group A beta-hemolytic streptococcal pharyngitis. None of these six cases were included in the data analysis to determine rates of environmentally responsive hypo or hyperthermia.

\section{External Temperatures}

The physical plant management staff at our hospital documents the external ambient air temperatures two to three times per day, in order to properly maintain the computer controlled indoor temperature. External temperature data from January 1992 through June 1993 was available for analysis for the purpose of this study. During the study period, the highest number of hypothermic events occurred in the month of June, with 13 documented events in 231 SCI patient days $(5.6 \%)$. The highest number of hyperthermic events during the study period occurred in the month of July, with four events recorded over 210 SCI patient days $(1.9 \%)$. The average temperature recorded for June 1992 and June 1993 was $68.3^{\circ}$ Fahrenheit (F), with a range of $51^{\circ}$ to $85^{\circ} \mathrm{F}$. The average temperature for July 1992 was $72^{\circ} \mathrm{F}$ with a range of $60^{\circ}$ to $90^{\circ} \mathrm{F}$.

\section{Discussion}

Normal thermoregulation requires an intact hypothalamus, brainstem and autonomic nervous system. $6,7,10,11$ The ability to shiver and increase basal metabolic rate in the face of cold exposure, and the ability to sweat and have perspiration condense on the surface of the skin, are necessary requirements for homeothermia. Vasodilation and vasoconstriction are also necessary for effective temperature modulation.

In patients with SCI, these thermoregulatory mechanisms are impaired to variable degrees. An inability to shiver below the level of the cord injury places the patient at risk for a core temperature decrement in the face of a prolonged cold exposure. Similarly, an inability to cutaneously vasodilate and/or sweat below the level of the spinal cord lesion makes dissipation of heat through evaporation of sweat on the skin impossible, and therefore leads to an unchecked increase in core temperature with a prolonged exposure to a high ambient temperature. Medications with autonomic effects such as bethanecol or beta-blockers may further impair the spinal cord injured persons' attempts at thermoregulation. ${ }^{9,13}$ Medications that alter peripheral vascular resistance through calcium channel blockade, can also complicate thermoregulation in the spinal cord injured person. ${ }^{4,13}$

The rate of environmentally induced hypothermia and hyperthermia in our population over 4059 SCI days was $1.60 \%$ and $0.34 \%$ respectively. This corresponds to the incidence of unexplained fevers previously reported in adults. ${ }^{6,7} \mathrm{We}$ were unable to find comparable data in the literature with respect to pediatric persons with SCI. As in the adult population, our results indicate that environmentally induced temperature instability appears to affect only a select subset of pediatric aged spinal cord injured persons. In our series, those most susceptible were newly injured patients with SCI, particularly those who had complete, high level cervical cord lesions and were ventilator dependent. Out of 79 total events, only two $(2.5 \%)$ resulted in temporary adverse patient outcomes, which reversed upon restoration of autonomic stability and normal core temperature. No significant mortality or irreversible morbidity was sustained as a result of any incident of hypo or hyperthermia. Moreover, delaying instigation of a 'rule-out sepsis' work-up until the subject's response to the temperature instability protocol was documented, did not appear to miss any significant infectious etiology for hypo or hyperthermia.

Our data indicate that during the study period, the highest rate of hypo and hyperthermic events occurred in June and July respectively. Average external temperatures during these months were $68.3^{\circ} \mathrm{F}$ and $72.0^{\circ} \mathrm{F}$. Despite the attempt at computer generated climate control in our institution, it was apparent from our study that the internal temperature adjustment often lagged behind the out of door shift in 
temperature, particularly in months of significant temperature fluctuation.

An unanticipated finding which resulted from our data collection was that the internal hospital room temperature could be falsely elevated or depressed, by as much as $10^{\circ} \mathrm{F}$, in cases where the thermostat had been inadvertently positioned adjacent to an outside window. This prompted our maintenance department, at the request of our Risk Management analyst, to adjust the location of the thermostat to the head of the bed in each room of the SCI unit.

As a result of our data collection and analysis, very specific recommendations are now in place on our SCI unit for the management of the environmental contribution to temperature instability in patients at risk. We recommend that in SCI patients with a level at T6 or above, that the room temperature be maintained between 72 to $74^{\circ} \mathrm{F}$, the temperature of the ventilator cascade be maintained between 30 to $32^{\circ} \mathrm{C}$, and clothing be adjusted as required for symptomatic episodes of temperature instability. Moreover, we have relocated the position of the thermostats in each of the patient rooms on our unit to a site above the head of the patient's bed.

Education of the entire inpatient rehabilitation team is essential in order to prevent environmentally induced temperature instability. If the designated nursing interventions fail to initiate the trend toward restoration of a normal body temperature, diagnostic interventions and a laboratory work-up is begun as appropriate.

Environmentally induced temperature instability is a relatively uncommon but easily managed occurrence in hospitalized, pediatric patients with SCI. Those patients at greatest risk are newly injured ventilator dependent persons with tetraplegia and associated brainstem injuries. Significant morbidity as a result of environmentally responsive hypo or hyperthermia is rare. In our patient series, the highest rate of occurrences for hypo and hyperthermia occurred in June and July respectively.

Awareness of the possibility of environmental influences on core temperature in SCI patients is critical for prevention and early recognition of this phenomenon. Appropriate location of thermostats in patient rooms so that temperature readings accurately reflect true ambient temperature at the patient's bedside is a simple, but effective physical modification of the environment designed to minimize the role of room temperature on patient temperature. Moreover, appropriate environmental manipulation by caregivers, through adjustment of ambient temperature, ventilator cascade temperature or clothing in this patient population may lower the economic burden caused by otherwise unnecessary laboratory investigations. The environmentally induced temperature instability protocol outlined in this paper is not intended to replace the need for medical assessment or investigation, but is designed to exclude an environmental contribution to temperature instability before an extensive medical work-up is begun.

\section{References}

1 Guttman L, Silver J, Wyndham $\mathrm{CH}$. Thermoregulation in spinal man. J Physiol 1958; 142: 406-419.

2 Bloch RF. Autonomic Dysfunction. In: Bloch and Basbaum (Eds) Management of Spinal Cord Injuries. The Williams and Wilkins Company: Baltimore 1986; pp 149-163.

3 Altus PA, Hickman JW, Nord HJ. Accidental hypothermia in a healthy quadriplegic patient. $J$ Neurology $1985 ; 35$ : 427-428.

4 Menard MR, Hahn G. Acute and chronic hypothermia in a man with spinal cord injury: environmental and pharmacologic causes. Arch Phys Med Rehabil 1991; 72: 421 - 424.

5 Sugarman B. Fever in recently injured quadriplegic persons. Arch Phys Med Rehabil 1982; 63: 639-640.

6 Beraldo PSS, Neves EGC, Alves CMF, Khan P, Cirilo ACM, Alencar MRB. Pyrexia in hospitalized pediatric patients. Paraplegia 1993; 31: $186-191$.

7 Sugarman B, Brown D, Musher D. Fever and infection in spinal cord injury patients. JAMA 1982; 248: $66-70$.

8 Colachis SC IIIrd, Otis SM. Occurrence of fever associated with thermoregulatory dysfunction after acute traumatic spinal cord injury. Am J Phys Rehabil 1995; 74: 114-119.

9 Berard EJJ, Bouchard MH, Depassio J, Fyon P. Effects of bethanecol and adreno-blockers on thermoregulation in spinal cord injury. Paraplegia 1989; 27: 46-50.

10 Schmidt KD, Chan CW. Thermoregulation and fever in normal persons and in those with spinal cord injuries. Mayo Clin Proc 1992; 67: 469-475.

11 Astrand PO, Rodahl K. Temperature regulation. In: Textbook of Work Physiology. $3^{\text {rd }}$ edn. McGraw Hill: New York 1986; pp $583-645$.

12 American Spinal Injury Association. International Standards for Neurological and Functional Classification of Spinal Cord Injury. Rev edn. The Association: Chicago 1996.

13 Bernheim HA, Block LH, Atkins E. Fever: Pathogenesis, pathophysiology and purpose. Annals Int Med 1979; 91: 261 270. 